\title{
Multiple solutions of boundary value problems with $\phi$-Laplacian operators and under a Wintner-Nagumo growth condition
}

\author{
Noha El Khattabi ${ }^{1}$, Marlene Frigon $^{2 *}$ and Nourredine Ayyadi ${ }^{1}$
}

Dedicated to Professor Jean Mawhin

${ }^{*}$ Correspondence:
frigon@dms.umontreal.ca
${ }^{2}$ Département de mathématiques
et de statistique, Université de
Montréal, succ. Centre-Ville, C.P.
6128, Montréal, H3C $3 J 7$, Canada
Full list of author information is
available at the end of the article

${ }^{*}$ Correspondence:

² épartement de mathématiques

et de statistique, Université de

Full list of author

a

\begin{abstract}
In this paper, we establish the existence of multiple solutions to second-order differential equations with $\phi$-Laplacian satisfying periodic, Dirichlet or Neumann boundary conditions. The right-hand side is a Carathéodory function satisfying a growth condition of Wintner-Nagumo type. The existence of upper and lower solutions is assumed. The proofs rely on the fixed point index theory.

MSC: Primary 34B15; secondary 34C25; 47H10; 37C25; 47H11
\end{abstract}

Keywords: boundary value problem; second-order differential equation; multiple solutions; $\phi$-Laplacian; upper and lower solutions; Wintner-Nagumo growth condition; fixed point index

\section{Introduction}

In this paper, we consider boundary value problems for second-order nonlinear differential equations with $\phi$-Laplacian of the form:

$$
\begin{aligned}
& \left(\phi\left(u^{\prime}(t)\right)\right)^{\prime}=f\left(t, u(t), u^{\prime}(t)\right) \text { a.e. } t \in[0, T], \\
& \quad u \in \mathcal{B},
\end{aligned}
$$

where $\mathcal{B}$ denotes the Dirichlet, periodic, or Neumann boundary conditions:

$$
\begin{aligned}
& u(0)=\mu, \quad u(T)=v ; \\
& u(0)=u(T), \quad \phi\left(u^{\prime}(0)\right)=\phi\left(u^{\prime}(T)\right) ; \\
& \phi\left(u^{\prime}(0)\right)=\mu, \quad \phi\left(u^{\prime}(T)\right)=v .
\end{aligned}
$$

Here, $f:[0, T] \times \mathbb{R}^{2} \rightarrow \mathbb{R}$ is a Carathéodory function, and $\phi: \mathbb{R} \rightarrow \mathbb{R}$ is a bijective increasing homeomorphism.

Such problems have been studied by many authors. The method of upper and lower solutions was widely used to obtain existence results; see, for instance, [1-16] and the references therein.

Many multiplicity results were obtained with the method of strict lower and upper solutions and for problems with the right member being a Carathéodory map not de-

\section{Springer}

๑2013 El Khattabi et al.; licensee Springer. This is an Open Access article distributed under the terms of the Creative Commons Attribution License (http://creativecommons.org/licenses/by/2.0), which permits unrestricted use, distribution, and reproduction in any medium, provided the original work is properly cited. 
pending on the derivative, $f(t, u)$. To our knowledge, De Coster [7] was the first to obtain multiplicity results for this problem with the Dirichlet boundary condition. Ben-Naoum and De Coster [1] considered the case where $\phi$ is the $p$-Laplacian with Sturm-Liouville boundary condition. Bereanu and Mawhin [2] established the existence of multiple periodic solutions in the case where $f(t, u)=e(t)-g(u)+c$ and $\phi$ can have a bounded domain or a bounded range. Zhang et al. [16] considered the periodic problem with the right member being a continuous map depending also on the derivative $f\left(t, u, u^{\prime}\right)$. A Nagumo growth condition was imposed on $f$. The case where $f\left(t, u, u^{\prime}\right)$ is a Carathéodory map and $\phi\left(u^{\prime}\right)=u^{\prime}$ was studied by El Khattabi [8] under a linear growth condition, and by Goudreau [11] under a Wintner-Nagumo growth condition.

Existence results were established for problem (1.1) under a growth condition of Nagumo type of the form:

$$
|f(t, x, y)| \leq \theta(|y|) k(t)
$$

with

$$
\min \left\{\int_{\phi(d)}^{\infty} \frac{\phi^{-1}(s)^{(p-1) / p}}{\theta\left(\phi^{-1}(s)\right)} d s, \int_{-\infty}^{\phi(-d)} \frac{\phi^{-1}(s)^{(p-1) / p}}{\theta\left(\phi^{-1}(s)\right)} d s\right\}>c,
$$

for $c, d$ suitable constants. In particular, the existence of a solution was obtained by O'Regan [13] when the right member has the form $q(t) f\left(t, u, u^{\prime}\right)$ with $f$ continuous, and with either Dirichlet or mixed boundary conditions. His result was extended in [14] and in [15] for a Carathéodory function $f$. Cabada and Pouso [3] considered also a Carathéodory $\operatorname{map} f$, and they established the existence of a solution to the problem with Neumann or periodic boundary conditions. More general boundary conditions or more general operators $\phi$ were considered in $[4-6,9,12]$. All those results rely on the Schauder fixed point theorem.

In this paper, we consider a Carathéodory map $f$ satisfying a growth condition different from (1.5). Namely, we impose the growth condition of Wintner-Nagumo type

$$
|f(t, x, y)| \leq \psi(|y|)\left(l(t)+c(t)|y|^{(p-1) / p}\right) .
$$

Using the method of upper and lower solutions and the fixed point index theory, we establish existence and multiplicity results for problem (1.1) with Dirichlet, Neumann or periodic boundary value conditions. Our proofs rely on the fixed point index theory. This theory is particularly convenient for the Neumann problem where we use the contraction property of the fixed point index to reduce the computation of the fixed point index in an affine space.

\section{Preliminaries}

In what follows, we denote $I=[0, T]$. The space of continuous functions $\mathcal{C}(I)$, and the space of continuously derivable functions $\mathcal{C}^{1}(I)$ are equipped with the usual norms $\|\cdot\|_{0}$ and $\|u\|_{1}=\max \left\{\|u\|_{0},\left\|u^{\prime}\right\|_{0}\right\}$, respectively. We denote the usual norm in $L^{p}(I)$ by $\|u\|_{L^{p}}$, where $1 \leq p \leq \infty$. We set

$$
W(I)=\left\{u \in \mathcal{C}^{1}(I): \phi\left(u^{\prime}\right) \text { is absolutely continuous and }\left(\phi\left(u^{\prime}\right)\right)^{\prime} \in L^{1}(I)\right\} .
$$


Definition 2.1 A map $f: I \times \mathbb{R}^{2} \rightarrow \mathbb{R}$ is a Carathéodory function if

(i) $f(t, \cdot, \cdot)$ is continuous for almost every $t \in I$;

(ii) $f(\cdot, x, y)$ is measurable for all $(x, y) \in \mathbb{R}^{2}$;

(iii) for all $R>0$, there exists $h_{R} \in L^{1}(I)$ such that $|f(t, x, y)| \leq h_{R}(t)$ for all $(x, y) \in \mathbb{R}^{2}$ such that $|x| \leq R,|y| \leq R$, and for almost every $t \in I$.

Definition 2.2 We say that $\alpha \in W(I)$ is a lower solution of (1.1) if

$$
\left(\phi\left(\alpha^{\prime}(t)\right)\right)^{\prime} \geq f\left(t, \alpha(t), \alpha^{\prime}(t)\right), \quad \text { a.e. } t \in I
$$

and, in addition,

(i) if $\mathcal{B}$ denotes Dirichlet boundary condition (1.2), it satisfies

$$
\alpha(0) \leq \mu \quad \text { and } \quad \alpha(T) \leq \nu ;
$$

(ii) if $\mathcal{B}$ denotes periodic boundary condition (1.3), it satisfies

$$
\alpha(0)=\alpha(T) \quad \text { and } \quad \phi\left(\alpha^{\prime}(0)\right) \geq \phi\left(\alpha^{\prime}(T)\right) ;
$$

(iii) if $\mathcal{B}$ denotes Neumann boundary condition (1.4), it satisfies

$$
\phi\left(\alpha^{\prime}(0)\right) \geq \mu \quad \text { and } \quad \phi\left(\alpha^{\prime}(T)\right) \leq \nu
$$

Similarly, we define an upper solution of (1.1) if the previous conditions are satisfied with the reversed inequalities.

Definition 2.3 We say that $\alpha \in W(I)$ is a strict lower solution of (1.1) if for any $\left.t_{0} \in\right] 0, T$, there exist $\varepsilon>0$ and $\mathcal{U}_{t_{0}}$, a neighborhood of $t_{0}$ in $I$, such that for almost every $t \in \mathcal{U}_{t_{0}}$ and all $(x, y) \in[\alpha(t), \alpha(t)+\varepsilon] \times\left[\alpha^{\prime}(t)-\varepsilon, \alpha^{\prime}(t)+\varepsilon\right]$,

$$
\left(\phi\left(\alpha^{\prime}(t)\right)\right)^{\prime} \geq f(t, x, y)
$$

and, in addition,

(i) if $\mathcal{B}$ denotes Dirichlet boundary condition (1.2), it satisfies

$$
\alpha(0)<\mu \quad \text { and } \quad \alpha(T)<\nu
$$

(ii) if $\mathcal{B}$ denotes periodic boundary condition (1.3), it satisfies

$$
\alpha(0)=\alpha(T) \quad \text { and } \quad \phi\left(\alpha^{\prime}(0)\right)>\phi\left(\alpha^{\prime}(T)\right) ;
$$

(iii) if $\mathcal{B}$ denotes Neumann boundary condition (1.4), it satisfies

$$
\phi\left(\alpha^{\prime}(0)\right)>\mu \quad \text { and } \quad \phi\left(\alpha^{\prime}(T)\right)<\nu .
$$

Similarly, we define a strict upper solution of (1.1) if the previous conditions are satisfied with the reversed inequalities. 
We will use the following general assumptions.

$\left(\mathrm{H}_{\phi}\right) \quad$ The map $\phi: \mathbb{R} \rightarrow \mathbb{R}$ is a bijective increasing homeomorphism.

$\left(\mathrm{H}_{f}\right) \quad$ The map $f: I \times \mathbb{R}^{2} \rightarrow \mathbb{R}$ is Carathéodory.

$\left(\mathrm{H}_{\mathcal{B}}\right)$ There exist $\alpha, \beta \in W(I)$, respectively lower and upper solutions of (1.1), such that $\alpha(t) \leq \beta(t)$ for all $t \in I$.

(WN) There exist $k>0, p \in] 1, \infty], c \in L^{p}\left(I,\left[0, \infty[), l \in L^{1}(I,[0, \infty[)\right.\right.$ and $\psi:[0, \infty[\rightarrow$ $[k, \infty[$ such that

$$
\frac{1}{\psi \circ \phi^{-1}} \in L_{l o c}^{1}(\mathbb{R}), \quad \int_{-\infty}^{\infty} \frac{d s}{\psi\left(\left|\phi^{-1}(s)\right|\right)}=\infty,
$$

and

$$
\begin{gathered}
|f(t, x, y)| \leq \psi(|y|)\left(l(t)+c(t)|y|^{(p-1) / p}\right) \\
\forall x \in[\alpha(t), \beta(t)], y \in \mathbb{R} \text {, and a.e. } t \in I,
\end{gathered}
$$

with $(p-1) / p=1$ if $p=\infty$.

In what follows, $\left(\mathrm{H}_{\mathcal{B}}\right)$ will be replaced by $\left(\mathrm{H}_{D}\right),\left(\mathrm{H}_{N}\right)$ or $\left(\mathrm{H}_{P}\right)$ if $\mathcal{B}$ denotes $(1.2),(1.3)$ or (1.4), the Dirichlet, periodic or Neumann boundary conditions, respectively.

We present some properties of operators that will be used later. Here is a particular case of Lemmas 2.3 and 2.6 in [10].

Lemma 2.4 Let $f: I \times \mathbb{R}^{2} \rightarrow \mathbb{R}$ be a Carathéodory function and $\sigma: I \times \mathbb{R}^{2} \rightarrow \mathbb{R}$ such that

(i) for any $u \in \mathcal{C}^{1}(I)$, the map $t \mapsto \sigma\left(t, u(t), u^{\prime}(t)\right)$ is measurable;

(ii) for any sequence $\left\{u_{n}\right\}$ converging to $u_{0}$ in $\mathcal{C}^{1}(I)$, there exists $c>0$ such that

$$
\left\|\sigma\left(t, u_{n}(t), u_{n}^{\prime}(t)\right)\right\| \leq c \quad \text { a.e. } t \in I \text { and } \forall n \geq 0
$$

and

$$
\sigma\left(t, u_{n}(t), u_{n}^{\prime}(t)\right) \rightarrow \sigma\left(t, u_{0}(t), u_{0}^{\prime}(t)\right) \quad \text { a.e. } t \in I
$$

Then the operator $N_{g}: \mathcal{C}^{1}(I) \rightarrow \mathcal{C}(I)$ defined by

$$
N_{g}(t)=\int_{0}^{t} g\left(s, u(s), u^{\prime}(s)\right) d s
$$

with

$$
g\left(t, u(t), u^{\prime}(t)\right)=f\left(t, \sigma\left(t, u(t), u^{\prime}(t)\right)\right),
$$

is continuous and completely continuous. Moreover, for every $u \in \mathcal{C}^{1}(I), N_{g}(u)$ is absolutely continuous and

$$
\frac{d}{d t} N_{g}(u)(t)=g\left(t, u(t), u^{\prime}(t)\right) \quad \text { a.e. } t \in I .
$$


Observe that the previous lemma holds with $\sigma(t, x, y)=(x, y)$. In this case, $N_{g}=N_{f}$.

Now, we present some results related to the homeomorphism $\phi$. The first one is a lemma due to Manásevich and Mawhin [17].

Lemma 2.5 Let $\phi: \mathbb{R} \rightarrow \mathbb{R}$ satisfy $\left(\mathrm{H}_{\phi}\right)$, and let $\mathcal{G}: \mathbb{R} \times \mathcal{C}(I) \rightarrow \mathbb{R}$ be defined by

$$
\mathcal{G}(a, h)=\int_{0}^{T} \phi^{-1}(a+h(t)) d t
$$

Then the following statements hold:

(1) For any $c \in \mathbb{R}$ and any $h \in \mathcal{C}(I)$, the equation

$$
\mathcal{G}(a, h)=c
$$

has a unique solution $\bar{a}_{c}(h)$.

(2) For any $c \in \mathbb{R}$, the function $\bar{a}_{c}: \mathcal{C}(I) \rightarrow \mathbb{R}$ defined in (1) is continuous, and it sends bounded sets into bounded sets.

It is easy to show the following result.

Lemma 2.6 Assume that $\phi: \mathbb{R} \rightarrow \mathbb{R}$ satisfies $\left(\mathrm{H}_{\phi}\right)$. Let $\Phi: \mathbb{R} \times \mathcal{C}(I) \rightarrow \mathcal{C}^{1}(I)$ be defined by

$$
\Phi(r, h)(t)=r+\int_{0}^{t} \phi^{-1}(h(s)) d s .
$$

Then $\Phi$ is continuous.

We will use the following maximum principle type result.

Lemma 2.7 Assume that $\left(\mathrm{H}_{\phi}\right)$ is satisfied. Let $v, w \in W(I)$ be such that

$$
\left(\phi\left(v^{\prime}(t)\right)\right)^{\prime} \leq\left(\phi\left(w^{\prime}(t)\right)\right)^{\prime} \quad \text { a.e. on }\{t \in I: v(t)<w(t)\} .
$$

Assume that one of the following conditions holds:

(i) $v(0) \geq w(0), v(T) \geq w(T)$;

(ii) $\phi\left(v^{\prime}(0)\right) \leq \phi\left(w^{\prime}(0)\right), \phi\left(v^{\prime}(T)\right) \geq \phi\left(w^{\prime}(T)\right)$;

(iii) $v(0)=v(T), w(0)=w(T), \phi\left(v^{\prime}(0)\right)-\phi\left(v^{\prime}(T)\right) \leq \phi\left(w^{\prime}(0)\right)-\phi\left(w^{\prime}(T)\right)$.

Then $v(t) \geq w(t)$ for all $t \in I$, or there exists $c>0$ such that $v(t)=w(t)-c$ for all $t \in I$.

Proof Assume that

$$
A=\{t \in I: v(t)<w(t)\} \neq \emptyset .
$$

Condition (i) (resp. (ii) or (iii)) implies that one of the following statements holds:

there exists $\left[t_{0}, t_{1}\right] \subset A$ such that

$$
v\left(t_{0}\right)-w\left(t_{0}\right)<v\left(t_{1}\right)-w\left(t_{1}\right) \text { and } \phi\left(v^{\prime}\left(t_{0}\right)\right) \leq \phi\left(w^{\prime}\left(t_{0}\right)\right) ;
$$


there exists $\left[t_{0}, t_{1}\right] \subset A$ such that

$v\left(t_{0}\right)-w\left(t_{0}\right)>v\left(t_{1}\right)-w\left(t_{1}\right)$ and $\phi\left(v^{\prime}\left(t_{1}\right)\right) \geq \phi\left(w^{\prime}\left(t_{1}\right)\right) ;$

or

there exists $c>0$ such that $\quad v(t)=w(t)-c \quad \forall t \in I$.

Indeed, if (2.5) does not hold, let $\tau, \rho \in I$ be such that

$$
v(\tau)-w(\tau)=\min \{v(t)-w(t): t \in I\}<v(\rho)-w(\rho),
$$

and

$v(t)-w(t)<0 \quad$ for all $t$ between $\tau$ and $\rho$.

If $\tau \in] 0, T$, then $\phi\left(v^{\prime}(\tau)\right)=\phi\left(w^{\prime}(\tau)\right)$ and (2.3) or (2.4) hold.

If $\tau \in\{0, T\}$, then (i) does not hold. If (ii) holds, then

$$
\phi\left(v^{\prime}(0)\right) \leq \phi\left(w^{\prime}(0)\right) \quad \text { and } \quad \phi\left(v^{\prime}(T)\right) \geq \phi\left(w^{\prime}(T)\right) .
$$

Similarly, if (iii) holds, since

$$
v(0)-w(0)=v(T)-w(T)=v(\tau)-w(\tau),
$$

one has $v^{\prime}(0) \geq w^{\prime}(0)$ and $v^{\prime}(T) \leq w^{\prime}(T)$. Using (iii) and the fact that $\phi$ is increasing, we get

$$
\phi\left(v^{\prime}(0)\right)-\phi\left(w^{\prime}(0)\right) \geq 0 \geq \phi\left(v^{\prime}(T)\right)-\phi\left(w^{\prime}(T)\right) \geq \phi\left(v^{\prime}(0)\right)-\phi\left(w^{\prime}(0)\right) .
$$

Hence, (2.3) or (2.4) are satisfied.

Therefore, by assumption,

$$
\left(\phi\left(v^{\prime}(t)\right)\right)^{\prime} \leq\left(\phi\left(w^{\prime}(t)\right)\right)^{\prime} \quad \text { a.e. } t \in\left[t_{0}, t_{1}\right] .
$$

If (2.3) holds, for every $\left.t \in] t_{0}, t_{1}\right]$,

$$
\begin{aligned}
\phi\left(v^{\prime}(t)\right)-\phi\left(v^{\prime}\left(t_{0}\right)\right) & =\int_{t_{0}}^{t}\left(\phi\left(v^{\prime}(s)\right)\right)^{\prime} d s \leq \int_{t_{0}}^{t}\left(\phi\left(w^{\prime}(s)\right)\right)^{\prime} d s \\
& =\phi\left(w^{\prime}(t)\right)-\phi\left(w^{\prime}\left(t_{0}\right)\right) \leq \phi\left(w^{\prime}(t)\right)-\phi\left(v^{\prime}\left(t_{0}\right)\right) .
\end{aligned}
$$

Since $\phi$ is increasing, we deduce that $v-w$ is nonincreasing in $\left[t_{0}, t_{1}\right]$. This is a contradiction. Similarly, we obtain a contradiction if (2.4) holds.

Therefore, $v(t) \geq w(t)$ for all $t \in I$, or (2.5) is satisfied.

Lemma 2.8 Assume $\left(\mathrm{H}_{\phi}\right)$ and $\left(\mathrm{H}_{f}\right)$. Let $\alpha, \beta \in W(I)$ be respectively strict lower and upper solutions of (1.1) such that $\alpha(t)<\beta(t)$ for all $t \in I$. If $u \in W(I)$ is a solution of (1.1) such that $\alpha(t) \leq u(t) \leq \beta(t)$ for all $t \in I$, then $\alpha(t)<u(t)<\beta(t)$ for all $t \in I$. 
Proof Let $u \in W(I)$ be a solution of (1.1) such that $\alpha(t) \leq u(t) \leq \beta(t)$ for all $t \in I$. Assume that

$$
A=\{t \in I: \alpha(t)=u(t)\} \neq \emptyset
$$

First, we claim that $0, T \notin A$. This is obviously the case if $\mathcal{B}$ denotes Dirichlet boundary condition (1.2). If $\mathcal{B}$ denotes periodic boundary condition (1.3), then $u-\alpha$ attains a minimum at 0 and $T$. So, by $\left(\mathrm{H}_{\phi}\right)$ and Definition 2.3(ii),

$$
\phi\left(u^{\prime}(T)\right)-\phi\left(\alpha^{\prime}(T)\right) \leq 0 \leq \phi\left(u^{\prime}(0)\right)-\phi\left(\alpha^{\prime}(0)\right)<\phi\left(u^{\prime}(T)\right)-\phi\left(\alpha^{\prime}(T)\right),
$$

a contradiction. If $\mathcal{B}$ denotes Neumann boundary condition (1.4), then $u-\alpha$ attains a minimum at 0 or at $T$. So, by $\left(\mathrm{H}_{\phi}\right)$ and Definition 2.3(iii),

$$
0 \leq \phi\left(u^{\prime}(0)\right)-\phi\left(\alpha^{\prime}(0)\right)<\mu-\mu \quad \text { or } \quad 0 \geq \phi\left(u^{\prime}(T)\right)-\phi\left(\alpha^{\prime}(T)\right)>v-v,
$$

a contradiction.

Let $\left.t_{0}=\max A \in\right] 0, T\left[\right.$. So, $\phi\left(u^{\prime}\left(t_{0}\right)\right)=\phi\left(\alpha^{\prime}\left(t_{0}\right)\right)$. By Definition 2.3, there exist $\varepsilon>0$ and $\mathcal{U}_{t_{0}}$, a neighborhood of $t_{0}$, such that $\left(\phi\left(\alpha^{\prime}(t)\right)\right)^{\prime} \geq f(t, x, y)$ a.e. $t \in \mathcal{U}_{t_{0}}$ and all $x, y$ such that $\alpha(t) \leq x \leq \alpha(t)+\varepsilon$ and $\left|y-\alpha^{\prime}(t)\right| \leq \varepsilon$. Since $u \in \mathcal{C}^{1}(I)$, there exists $\left.t_{1} \in\right] t_{0}, T$ [ such that $u(t) \in] \alpha(t), \alpha(t)+\varepsilon]$ and $\left|u^{\prime}(t)-\alpha^{\prime}(t)\right| \leq \varepsilon$ for all $\left.\left.t \in\right] t_{0}, t_{1}\right]$. Since $t_{0}=\max A$, there exists $\left.t \in] t_{0}, t_{1}\right]$ such that $u^{\prime}(t)>\alpha^{\prime}(t)$. Using the fact that $\phi$ is increasing, we deduce that

$$
\begin{aligned}
\phi\left(\alpha^{\prime}(t)\right)-\phi\left(\alpha^{\prime}\left(t_{0}\right)\right) & <\phi\left(u^{\prime}(t)\right)-\phi\left(u^{\prime}\left(t_{0}\right)\right)=\int_{t_{0}}^{t} f\left(s, u(s), u^{\prime}(s)\right) d s \\
& \leq \int_{t_{0}}^{t}\left(\phi\left(\alpha^{\prime}(s)\right)\right)^{\prime} d s=\phi\left(\alpha^{\prime}(t)\right)-\phi\left(\alpha^{\prime}\left(t_{0}\right)\right) .
\end{aligned}
$$

This is a contradiction. Therefore, $\alpha(t)<u(t)$ for all $t \in I$.

Similarly, we show that $\beta(t)>u(t)$ for all $t \in I$.

The following result establishes the existence of an a priori bound on the derivative of functions satisfying a suitable inequality.

Lemma 2.9 Assume that $\left(\mathrm{H}_{\phi}\right)$ is satisfied. Let $k>0$ and $\psi:[0, \infty[\rightarrow[k, \infty[$ be such that

$$
\frac{1}{\psi \circ \phi^{-1}} \in L_{l o c}^{1}(\mathbb{R}) \text { and } \int_{0}^{ \pm \infty} \frac{d s}{\psi\left(\left|\phi^{-1}(s)\right|\right)}= \pm \infty \text {. }
$$

Then, for every $\left.\left.d_{0} \geq 0, p \in\right] 1, \infty\right], c_{0} \in L^{p}\left(I,\left[0, \infty[), l_{0} \in L^{1}(I,[0, \infty[)\right.\right.$, and $B \subset \mathcal{C}(I)$ bounded, there exists $M_{0}>d_{0}$ such that for every

$$
u \in\left\{u \in W(I): u \in B, \min \left\{\left|u^{\prime}(t)\right|: t \in I\right\} \leq d_{0}\right\}
$$

satisfying

$$
\left|\left(\phi\left(u^{\prime}(t)\right)\right)^{\prime}\right| \leq \psi\left(\left|u^{\prime}(t)\right|\right)\left(l_{0}(t)+c_{0}(t)\left|u^{\prime}(t)\right|^{(p-1) / p}\right) \quad \text { a.e. } t \in I,
$$

one has $\left\|u^{\prime}\right\|_{0}<M_{0}$. 
Proof Let

$$
m=\max \left\{u\left(t_{1}\right)-u\left(t_{2}\right): t_{1}, t_{2} \in I, u \in B\right\} .
$$

Assumptions $\left(\mathrm{H}_{\phi}\right)$ and (2.7) imply that there exists $M_{0}>d_{0}$ such that

$$
\phi\left(M_{0}\right)>\phi\left(d_{0}\right) \geq \phi\left(-d_{0}\right)>\phi\left(-M_{0}\right)
$$

and

$$
\begin{aligned}
& \max \left\{\int_{\phi\left(d_{0}\right)}^{\phi\left(M_{0}\right)} \frac{d s}{\psi\left(\left|\phi^{-1}(s)\right|\right)}, \int_{\phi\left(-M_{0}\right)}^{\phi\left(-d_{0}\right)} \frac{d s}{\psi\left(\left|\phi^{-1}(s)\right|\right)}\right\} \\
& >\left\|l_{0}\right\|_{L^{1}}+m^{(p-1) / p}\left\|c_{0}\right\|_{L^{p}} .
\end{aligned}
$$

Assume that there exists

$$
\begin{aligned}
& u \in\left\{u \in W(I): u \in B, \min \left\{\left|u^{\prime}(t)\right|: t \in I\right\} \leq d_{0},\right. \\
& \left.\quad \text { and }\left|\left(\phi\left(u^{\prime}(t)\right)\right)^{\prime}\right| \leq \psi\left(\left|u^{\prime}(t)\right|\right)\left(l_{0}(t)+c_{0}(t)\left|u^{\prime}(t)\right|^{(p-1) / p}\right) \text { a.e. } t \in I\right\}
\end{aligned}
$$

such that $\left\|u^{\prime}\right\|_{0} \geq M_{0}$. If $\max \left\{u^{\prime}(t): t \in I\right\} \geq M_{0}$, there exist $t_{0}, t_{1} \in I$ such that $\phi\left(u^{\prime}\left(t_{0}\right)\right)=$ $\left.\phi\left(d_{0}\right), \phi\left(u^{\prime}\left(t_{1}\right)\right)=\phi\left(M_{0}\right), \phi\left(u^{\prime}(t)\right) \in\right] \phi\left(d_{0}\right), \phi\left(M_{0}\right)\left[\right.$ and $u^{\prime}(t)>0$ for all $t$ between $t_{0}$ and $t_{1}$. Without loss of generality, we assume that $t_{0}<t_{1}$. Then, by assumption,

$$
\frac{\left(\phi\left(u^{\prime}(t)\right)\right)^{\prime}}{\psi\left(u^{\prime}(t)\right)} \leq l_{0}(t)+c_{0}(t)\left(u^{\prime}(t)\right)^{(p-1) / p} \quad \text { a.e. } t \in\left[t_{0}, t_{1}\right] .
$$

Integrating from $t_{0}$ to $t_{1}$ and using the Hölder inequality and the change of variable formula in an integral give us

$$
\begin{aligned}
\int_{\phi\left(d_{0}\right)}^{\phi\left(M_{0}\right)} \frac{d s}{\psi\left(\left|\phi^{-1}(s)\right|\right)} & =\int_{t_{0}}^{t_{1}} \frac{\left(\phi\left(u^{\prime}(t)\right)\right)^{\prime}}{\psi\left(u^{\prime}(t)\right)} d t \\
& \leq \int_{t_{0}}^{t_{1}} l_{0}(t)+c_{0}(t)\left(u^{\prime}(t)\right)^{(p-1) / p} d t \\
& \leq\left\|l_{0}\right\|_{L^{1}}+\left\|c_{0}\right\|_{L^{p}}\left(u\left(t_{1}\right)-u\left(t_{0}\right)\right)^{(p-1) / p} \\
& \leq\left\|l_{0}\right\|_{L^{1}}+m^{(p-1) / p}\left\|c_{0}\right\|_{L^{p}} .
\end{aligned}
$$

This contradicts (2.8).

Similarly, if $\min \left\{u^{\prime}(t): t \in I\right\} \leq-M_{0}$, there exist $t_{0}, t_{1} \in I$ such that $\phi\left(u^{\prime}\left(t_{0}\right)\right)=\phi\left(-d_{0}\right)$, $\left.\phi\left(u^{\prime}\left(t_{1}\right)\right)=\phi\left(-M_{0}\right), \phi\left(u^{\prime}(t)\right) \in\right] \phi\left(-M_{0}\right), \phi\left(-d_{0}\right)\left[\right.$ and $u^{\prime}(t)<0$ for all $t$ between $t_{0}$ and $t_{1}$. Arguing as above leads to a contradiction.

\section{The Dirichlet problem}

In this section, we consider problem (1.1) with the Dirichlet boundary condition. In order to establish the existence of a solution to (1.1), (1.2), we consider the following family of 
problems defined for $\lambda \in[0,1]$ :

$$
\begin{aligned}
& \left(\phi\left(u^{\prime}(t)\right)\right)^{\prime}=\lambda \widehat{f}\left(t, u(t), u^{\prime}(t)\right), \quad \text { a.e. } t \in I, \\
& u(0)=\mu, \quad u(T)=v,
\end{aligned}
$$

where $\widehat{f}:[0,1] \times \mathbb{R}^{2} \rightarrow \mathbb{R}$ is defined by

$$
\widehat{f}(t, x, y)= \begin{cases}f\left(t, \beta(t), \beta^{\prime}(t)\right)-\widehat{h}(t)(x-\beta(t)) & \text { if } x>\beta(t) \\ f(t, x, y) & \text { if } \alpha(t) \leq x \leq \beta(t) \\ f\left(t, \alpha(t), \alpha^{\prime}(t)\right)+\widehat{g}(t)(x-\alpha(t)) & \text { if } x<\alpha(t)\end{cases}
$$

with $\widehat{g}, \widehat{h} \in L^{1}(I)$ chosen such that

$$
\widehat{g}(t)>\max \left\{0, f\left(t, \alpha(t), \alpha^{\prime}(t)\right)\right\} \quad \text { and } \quad \widehat{h}(t)<\min \left\{0, f\left(t, \beta(t), \beta^{\prime}(t)\right)\right\} .
$$

We show that the solutions to these problems are a priori bounded.

Proposition 3.1 Assume that $\left(\mathrm{H}_{\phi}\right),\left(\mathrm{H}_{f}\right),\left(\mathrm{H}_{D}\right)$ and $(\mathrm{WN})$ hold. Then there exists $M>$ $\max \left\{\|\alpha\|_{0},\|\beta\|_{0}\right\}$ such that any solution $u$ of $\left(3.1_{\lambda}\right)$ satisfies $\|u\|_{1}<M$.

Proof Fix $M_{1}>0$ such that

$$
-M_{1} \leq \min _{t \in I} \alpha(t)-1 \quad \text { and } \quad M_{1} \geq \max _{t \in I} \beta(t)+1 .
$$

We claim that any solution $u$ of $\left(3.1_{\lambda}\right)$ is such that $\|u\|_{0} \leq M_{1}$. Indeed, by $\left(\mathrm{H}_{D}\right)$,

$$
-M_{1}<u(0)=\mu<M_{1} \quad \text { and } \quad-M_{1}<u(T)=v<M_{1} .
$$

From the definition of $\widehat{f}$, one has, almost everywhere on $\left\{t \in I: u(t)<-M_{1}\right\}$,

$$
\begin{aligned}
\left(\phi\left(u^{\prime}(t)\right)\right)^{\prime} & =\lambda\left(f\left(t, \alpha(t), \alpha^{\prime}(t)\right)+\widehat{g}(t)(u(t)-\alpha(t))\right) \\
& \leq \lambda\left(f\left(t, \alpha(t), \alpha^{\prime}(t)\right)-\widehat{g}(t)\right) \\
& \leq 0
\end{aligned}
$$

Similarly,

$$
\left(\phi\left(u^{\prime}(t)\right)\right)^{\prime} \geq 0 \quad \text { a.e. on }\left\{t \in I: u(t)>M_{1}\right\}
$$

It follows from Lemma 2.7 that $-M_{1} \leq u(t) \leq M_{1}$ for all $t \in I$.

We look for an a priori bound on the derivative of any solution $u$ of $\left(3.1_{\lambda}\right)$. Let

$$
l_{1}(t)=\max \left\{\left|f\left(t, \alpha(t), \alpha^{\prime}(t)\right)\right|+2 M_{1}|\widehat{g}(t)|,\left|f\left(t, \beta(t), \beta^{\prime}(t)\right)\right|+2 M_{1}|\widehat{h}(t)|\right\} .
$$


Observe that, by $(\mathrm{WN})$,

$$
\begin{aligned}
\left|\left(\phi\left(u^{\prime}(t)\right)\right)^{\prime}\right| & \leq \max \left\{l_{1}(t), \psi\left(\left|u^{\prime}(t)\right|\right)\left(l(t)+c(t)\left|u^{\prime}(t)\right|^{(p-1) / p}\right)\right\} \\
& \leq \psi\left(\left|u^{\prime}(t)\right|\right)\left(l_{0}(t)+c(t)\left|u^{\prime}(t)\right|^{(p-1) / p}\right) \text { a.e. } t \in I,
\end{aligned}
$$

with

$$
l_{0}(t)=\max \left\{l(t), \frac{l_{1}(t)}{k}\right\} .
$$

It follows from Lemma 2.9 applied with $d_{0}=(\nu-\mu) / T$ that there exists $M_{0}$ such that any solution $u$ of $\left(3.1_{\lambda}\right)$ satisfies $\left\|u^{\prime}\right\|_{0}<M_{0}$.

Finally, set $M=\max \left\{M_{0}, 1+M_{1}\right\}$. We have that $\|u\|_{1}<M$ for any solution $u$ of $\left(3.1_{\lambda}\right)$.

Proposition 3.2 Assume that $\left(\mathrm{H}_{\phi}\right),\left(\mathrm{H}_{f}\right),\left(\mathrm{H}_{D}\right)$ and $(\mathrm{WN})$ hold. Then, for every $\lambda \in[0,1]$, problem $\left(3.1_{\lambda}\right)$ has at least one solution.

Proof Let us define $N_{\hat{f}}: \mathcal{C}^{1}(I) \rightarrow \mathcal{C}(I)$ and $\mathcal{S}_{D}:[0,1] \times \mathcal{C}^{1}(I) \rightarrow \mathbb{R} \times \mathcal{C}(I)$ by

$$
N_{\hat{f}}(u)(t)=\int_{0}^{t} \widehat{f}\left(s, u(s), u^{\prime}(s)\right) d s,
$$

and

$$
\mathcal{S}_{D}(\lambda, u)=\left(\mu, \bar{a}_{\nu-\mu}\left(\lambda N_{\hat{f}}(u)\right)+\lambda N_{\hat{f}}(u)\right)
$$

where $\bar{a}_{\nu-\mu}$ is obtained in Lemma 2.5. Now, we define $\mathcal{D}:[0,1] \times \mathcal{C}^{1}(I) \rightarrow \mathcal{C}^{1}(I)$ by

$$
\mathcal{D}=\Phi \circ \mathcal{S}_{D}
$$

where $\Phi$ is defined in Lemma 2.6. We deduce that $\mathcal{S}_{D}$ is continuous and completely continuous from Lemma 2.5 and from Lemma 2.4 applied with

$$
\sigma(t, x, y)= \begin{cases}\left(\beta(t), \beta^{\prime}(t)\right) & \text { if } x>\beta(t), \\ (x, y) & \text { if } \alpha(t) \leq x \leq \beta(t), \\ \left(\alpha(t), \alpha^{\prime}(t)\right) & \text { if } x<\alpha(t) .\end{cases}
$$

This combined with Lemma 2.6 implies that $\mathcal{D}$ is continuous and completely continuous.

Now, we study the fixed points of $\mathcal{D}$. Let $u \in \mathcal{C}^{1}(I)$ and $\lambda \in[0,1]$ be such that $u=\mathcal{D}(\lambda, u)$. One has, by Lemma 2.5,

$$
u(0)=\mu, \quad u(T)=\mu+\mathcal{G}\left(\bar{a}_{v-\mu}\left(\lambda N_{\hat{f}}(u)\right), \lambda N_{\hat{f}}(u)\right)=\mu+(v-\mu)=v .
$$

Also, $\phi\left(u^{\prime}\right)=\bar{a}_{\nu-\mu}\left(\lambda N_{\hat{f}}(u)\right)+\lambda N_{\hat{f}}(u)$. Hence, by Lemma 2.4 , it is absolutely continuous and

$$
\left(\phi\left(u^{\prime}(t)\right)\right)^{\prime}=\lambda \widehat{f}\left(t, u(t), u^{\prime}(t)\right) \quad \text { a.e. } t \in I .
$$

So, fixed points of $\mathcal{D}$ are solutions of $\left(3.1_{\lambda}\right)$. 
Let $M>\max \left\{\|\alpha\|_{0},\|\beta\|_{0}\right\} \geq \max \{\mu, \nu\}$ be the constant obtained in Proposition 3.1 and set

$$
\mathcal{U}=\left\{u \in \mathcal{C}^{1}(I):\|u\|_{1}<M\right\}
$$

Proposition 3.1 implies that $u \neq \mathcal{D}(\lambda, u)$ for all $(\lambda, u) \in[0,1] \times \partial \mathcal{U}$. Observe that $\mathcal{D}(0, u)=$ $\Phi\left(\mu, \bar{a}_{\nu-\mu}(0)\right)=u_{0}$ with $u_{0}(t)=\mu+(\nu-\mu) t / T$. One has $u_{0} \in \mathcal{U}$. By the properties of the fixed point index (see [18] for more details),

$$
\operatorname{index}(\mathcal{D}(\lambda, \cdot), \mathcal{U})=\operatorname{index}(\mathcal{D}(0, \cdot), \mathcal{U})=\operatorname{index}\left(u_{0}, \mathcal{U}\right)=1 \quad \forall \lambda \in[0,1]
$$

Therefore, for every $\lambda \in[0,1], \mathcal{D}(\lambda, \cdot)$ has a fixed point, and hence $\left(3.1_{\lambda}\right)$ has a solution.

Now, we can establish the existence of a solution to (1.1), (1.2).

Theorem 3.3 Assume that $\left(\mathrm{H}_{\phi}\right),\left(\mathrm{H}_{f}\right),\left(\mathrm{H}_{D}\right)$ and $(\mathrm{WN})$ hold. Then Dirichlet problem (1.1), (1.2) has a solution $u \in W(I)$ such that $\alpha(t) \leq u(t) \leq \beta(t)$ for every $t \in I$.

Proof Proposition 3.2 insures the existence of $u \in W(I)$, a solution of $\left(3.1_{\lambda}\right)$ for $\lambda=1$. To conclude, we have to show that $\alpha(t) \leq u(t) \leq \beta(t)$ for all $t \in I$ since $\widehat{f}(t, x, y)=f(t, x, y)$ for $x \in[\alpha(t), \beta(t)]$.

By $\left(\mathrm{H}_{D}\right)$,

$$
\begin{aligned}
& \left(\phi\left(u^{\prime}(t)\right)\right)^{\prime}=f\left(t, \alpha(t), \alpha^{\prime}(t)\right)+\widehat{g}(t)(x(t)-\alpha(t)) \leq f\left(t, \alpha(t), \alpha^{\prime}(t)\right) \\
& \leq\left(\phi\left(\alpha^{\prime}(t)\right)\right)^{\prime} \quad \text { a.e. } t \in\{t \in I: u(t)<\alpha(t)\}, \\
& u(0)=\mu \geq \alpha(0) \text { and } u(T)=v \geq \alpha(T) .
\end{aligned}
$$

It follows from Lemma 2.7 that $u(t) \geq \alpha(t)$ for all $t \in I$.

A similar argument yields $u(t) \leq \beta(t)$ for all $t \in I$.

Remark 3.4 The hypothesis (WN) can be generalized by

$$
\min \left\{\int_{\phi\left(d_{0}\right)}^{\infty} \frac{d s}{\psi\left(\left|\phi^{-1}(s)\right|\right)}, \int_{-\infty}^{\phi\left(-d_{0}\right)} \frac{d s}{\psi\left(\left|\phi^{-1}(s)\right|\right)}\right\}>c
$$

with $d_{0}=(v-\mu) / T$ and $c$, a suitable constant which can be deduced from the proof of Lemma 2.9 .

Example 3.5 Let us consider the following problem:

$$
\begin{aligned}
& \left(\phi\left(u^{\prime}(t)\right)\right)^{\prime}=\left(\left(u^{\prime}(t)\right)^{3}+1\right)\left(h(t)(u(t)+a(t))+g(u(t)) u^{\prime}(t)\right) \quad \text { a.e. } t \in[0, T], \\
& u(0)=0, \quad u(T)=0,
\end{aligned}
$$

where $\phi(s)=s^{3}, a \in C[0, T], g \in C(\mathbb{R})$ and $h \in L^{1}[0, T]$ with $h(t) \geq 0$ a.e. $t \in[0, T]$. Let $m>0$ be such that $|a(t)| \leq m$ for all $t \in T$. Then $\alpha=-m$ and $\beta=m$ are respectively lower 
and upper solutions of (3.10). Let $\psi(s)=\left(|s|^{3}+1\right), c \geq \max \{|g(x)|:|x| \leq m\}$ and $l=2 m h$. One has

$$
|f(t, x, y)| \leq \psi(|y|)(l(t)+c|y|) \quad \forall x \in[-m, m], y \in \mathbb{R} \text {, and a.e. } t \in[0, T],
$$

and

$$
\int_{-\infty}^{\infty} \frac{d s}{\psi\left(\left|\phi^{-1}(s)\right|\right)}=\int_{-\infty}^{\infty} \frac{d s}{|s|+1}=\infty
$$

By Theorem 3.3, (3.10) has a solution $u$ such that $|u(t)| \leq m$ for all $t \in[0, T]$. Notice that $f$ does not satisfy a growth condition of Nagumo type.

\section{The periodic problem}

In this section, we consider problem (1.1) with the periodic boundary condition. In order to establish the existence of a solution to (1.1), (1.3), we consider the following family of problems defined for $\lambda \in[0,1]$ :

$$
\begin{aligned}
& \left(\phi\left(u^{\prime}(t)\right)\right)^{\prime}=\lambda \widehat{f}\left(t, u(t), u^{\prime}(t)\right)+\frac{(1-\lambda)}{T} \int_{0}^{T} \widehat{f}\left(t, u(t), u^{\prime}(t)\right) d t \quad \text { a.e. } t \in I, \\
& u(0)=u(T), \quad \phi\left(u^{\prime}(0)\right)=\phi\left(u^{\prime}(T)\right),
\end{aligned}
$$

where $\widehat{f}$ is defined in (3.2).

We show that the solutions to these problems are a priori bounded.

Proposition 4.1 Assume that $\left(\mathrm{H}_{\phi}\right),\left(\mathrm{H}_{f}\right),\left(\mathrm{H}_{P}\right)$ and $(\mathrm{WN})$ hold. Then there exists $M>$ $\max \left\{\|\alpha\|_{0},\|\beta\|_{0}\right\}$ such that any solution $u$ of $\left(4.1_{\lambda}\right)$ satisfies $\|u\|_{1}<M$.

Proof Fix $M_{1}>0$ such that

$$
-M_{1} \leq \min _{t \in I} \alpha(t)-1 \quad \text { and } \quad M_{1} \geq \max _{t \in I} \beta(t)+1 .
$$

We claim that any solution $u$ of $\left(4.1_{\lambda}\right)$ is such that $\|u\|_{0} \leq M_{1}$. Observe that a solution of $\left(4.1_{\lambda}\right)$ satisfies

$$
\begin{aligned}
0 & =\phi\left(u^{\prime}(T)\right)-\phi\left(u^{\prime}(0)\right)=\lambda \int_{0}^{T} \widehat{f}\left(t, u(t), u^{\prime}(t)\right) d t+(1-\lambda) \int_{0}^{T} \widehat{f}\left(t, u(t), u^{\prime}(t)\right) d t \\
& =\int_{0}^{T} \widehat{f}\left(t, u(t), u^{\prime}(t)\right) d t .
\end{aligned}
$$

So, it satisfies

$$
\left(\phi\left(u^{\prime}(t)\right)\right)^{\prime}=\lambda \widehat{f}\left(t, u(t), u^{\prime}(t)\right) \quad \text { a.e. } t \in I
$$

with periodic boundary condition (1.3). Arguing as in the proof of Proposition 3.1, we obtain that

$$
\begin{array}{ll}
\left(\phi\left(u^{\prime}(t)\right)\right)^{\prime} \leq 0 & \text { a.e. on }\left\{t \in I: u(t)<-M_{1}\right\}, \\
\left(\phi\left(u^{\prime}(t)\right)\right)^{\prime} \geq 0 & \text { a.e. on }\left\{t \in I: u(t)>M_{1}\right\} .
\end{array}
$$


It follows from Lemma 2.7 that $\|u\|_{0} \leq M_{1}$, or there exists $c>0$ such that $|u(t)|=M_{1}+c$ for all $t \in I$. If $u \equiv M_{1}+c$,

$$
0=\int_{0}^{T} \widehat{f}\left(t, u(t), u^{\prime}(t)\right) d t=\int_{0}^{T} f\left(t, \beta(t), \beta^{\prime}(t)\right)-\widehat{h}(t)\left(M_{1}+c-\beta(t)\right) d t>0,
$$

a contradiction. Similarly, one cannot have $u \equiv-\left(M_{1}+c\right)$. Hence, $\|u\|_{0} \leq M_{1}$.

As in the proof of Proposition 3.1, one has that any solution $u$ of $\left(4.1_{\lambda}\right)$ satisfies

$$
\left|\left(\phi\left(u^{\prime}(t)\right)\right)^{\prime}\right| \leq \psi\left(\left|u^{\prime}(t)\right|\right)\left(l_{0}(t)+c(t)\left|u^{\prime}(t)\right|^{(p-1) / p}\right) \quad \text { a.e. } t \in I
$$

where $l_{0}$ is defined in (3.4). It follows from Lemma 2.9 applied with $d_{0}=0$ that there exists $M_{0}$ such that any solution $u$ of $\left(4.1_{\lambda}\right)$ satisfies $\left\|u^{\prime}\right\|_{0}<M_{0}$.

Finally, set $M=\max \left\{M_{0}, 1+M_{1}\right\}$. We have that $\|u\|_{1}<M$ for any solution $u$ of $\left(4 \cdot 1_{\lambda}\right)$.

Proposition 4.2 Assume that $\left(\mathrm{H}_{\phi}\right),\left(\mathrm{H}_{f}\right),\left(\mathrm{H}_{P}\right)$ and $(\mathrm{WN})$ hold. Then, for every $\lambda \in[0,1]$, problem $\left(4.1_{\lambda}\right)$ has at least one solution.

Proof Let $N_{\hat{f}}: \mathcal{C}^{1}(I) \rightarrow \mathcal{C}(I)$ be defined in (3.5). Let us consider the operators $\widehat{L}: \mathcal{C}^{1}(I) \rightarrow$ $\mathcal{C}(I)$ and $\mathcal{S}_{P}:[0,1] \times \mathcal{C}^{1}(I) \rightarrow \mathbb{R} \times \mathcal{C}(I)$ defined by

$$
\widehat{L}(u)(t)=\frac{t}{T} N_{\hat{f}}(u)(T),
$$

and

$$
\mathcal{S}_{P}(\lambda, u)=\left(u(0)+\frac{1}{T} N_{\hat{f}}(u)(T), \bar{a}_{0}\left(\lambda\left(N_{\hat{f}}(u)-\widehat{L}(u)\right)\right)+\lambda\left(N_{\hat{f}}(u)-\widehat{L}(u)\right)\right),
$$

where $\bar{a}_{0}$ is obtained in Lemma 2.5 . Now, we define $\mathcal{P}:[0,1] \times \mathcal{C}^{1}(I) \rightarrow \mathcal{C}^{1}(I)$ by

$$
\mathcal{P}=\Phi \circ \mathcal{S}_{P}
$$

Again, we deduce from Lemmas 2.4, 2.5 and 2.6 that $\mathcal{S}_{P}$ and $\mathcal{P}$ are continuous and completely continuous.

Now, we study the fixed points of $\mathcal{P}$. Let $u \in \mathcal{C}^{1}(I)$ and $\lambda \in[0,1]$ be such that $u=\mathcal{P}(\lambda, u)$. One has, by Lemma 2.5,

$$
\begin{aligned}
u(0) & =u(0)+\frac{1}{T} N_{\hat{f}}(u)(T), \\
u(T) & =u(0)+\frac{1}{T} N_{\hat{f}}(u)(T)+\mathcal{G}\left(\bar{a}_{0}\left(\lambda\left(N_{\hat{f}}(u)-\widehat{L}(u)\right)\right), \lambda\left(N_{\hat{f}}(u)-\widehat{L}(u)\right)\right) \\
& =u(0) .
\end{aligned}
$$

Notice that $\left(N_{\hat{f}}(u)(T)\right) / T=0$. Also,

$$
\phi\left(u^{\prime}\right)=\bar{a}_{0}\left(\lambda\left(N_{\hat{f}}(u)-\widehat{L}(u)\right)\right)+\lambda\left(N_{\hat{f}}(u)-\widehat{L}(u)\right) .
$$


Hence, by Lemma 2.4, it is absolutely continuous and

$$
\begin{aligned}
\left(\phi\left(u^{\prime}(t)\right)\right)^{\prime} & =\lambda\left(\widehat{f}\left(t, u(t), u^{\prime}(t)\right)-\frac{1}{T} N_{\hat{f}}(u)(T)\right) \\
& =\lambda \widehat{f}\left(t, u(t), u^{\prime}(t)\right)+\frac{(1-\lambda)}{T} \int_{0}^{T} \widehat{f}\left(t, u(t), u^{\prime}(t)\right) d t \quad \text { a.e. } t \in I .
\end{aligned}
$$

Moreover,

$$
\begin{aligned}
\phi\left(u^{\prime}(0)\right) & =\bar{a}_{0}\left(\lambda\left(N_{\hat{f}}(u)-\widehat{L}(u)\right)\right)+\lambda\left(N_{\hat{f}}(u)(0)-\widehat{L}(u)(0)\right), \\
\phi\left(u^{\prime}(T)\right) & =\bar{a}_{0}\left(\lambda\left(N_{\hat{f}}(u)-\widehat{L}(u)\right)\right)+\lambda\left(N_{\hat{f}}(u)(T)-\widehat{L}(u)(T)\right) \\
& =\phi\left(u^{\prime}(0)\right) .
\end{aligned}
$$

So, fixed points of $\mathcal{P}$ are solutions of $\left(4 \cdot 1_{\lambda}\right)$.

Let $M>\max \left\{\|\alpha\|_{0},\|\beta\|_{0}\right\}$ be the constant obtained in Proposition 4.1 and set

$$
\mathcal{U}=\left\{u \in \mathcal{C}^{1}(I):\|u\|_{1}<M\right\}
$$

Proposition 4.1 implies that $u \neq \mathcal{P}(\lambda, u)$ for all $(\lambda, u) \in[0,1] \times \partial \mathcal{U}$. By the homotopy property of the fixed point index,

$$
\operatorname{index}(\mathcal{P}(\lambda, \cdot), \mathcal{U})=\operatorname{index}(\mathcal{P}(0, \cdot), \mathcal{U}) \quad \forall \lambda \in[0,1]
$$

Observe that

$$
\mathcal{P}(0, u)=\Phi\left(u(0)+\frac{1}{T} N_{\hat{f}}(u)(T), \bar{a}_{0}(0)\right)=u(0)+\frac{1}{T} N_{\hat{f}}(u)(T) \in \mathbb{R}
$$

One has $\partial(\mathcal{U} \cap \mathbb{R})=\{-M, M\}$, and

$$
\begin{aligned}
\mathcal{P}(0, M) & =M+\frac{1}{T} \int_{0}^{T} \widehat{f}(t, M, 0) d t \\
& =M+\frac{1}{T} \int_{0}^{T} f\left(t, \beta(t), \beta^{\prime}(t)\right)-\widehat{h}(t)(M-\beta(t)) d t \\
& >M,
\end{aligned}
$$

since $M>\|\beta\|_{0}$ and $\widehat{h}(t)<\min \left\{0, f\left(t, \beta(t), \beta^{\prime}(t)\right)\right\}$. Similarly,

$$
\mathcal{P}(0,-M)<-M
$$

By the contraction property of the fixed point index (see [18, Chapter 4 , Section 12 , Theorem 6.2]),

$$
\operatorname{index}(\mathcal{P}(0, \cdot), \mathcal{U})=\operatorname{index}(\mathcal{P}(0, \cdot),(\mathcal{U} \cap \mathbb{R}))=-1
$$


Therefore, for every $\lambda \in[0,1]$,

$$
\operatorname{index}(\mathcal{P}(\lambda, \cdot), \mathcal{U})=-1
$$

Thus, $\mathcal{P}(\lambda, \cdot)$ has a fixed point, and hence $\left(4.1_{\lambda}\right)$ has a solution.

Now, we can establish the existence of a solution to (1.1), (1.3).

Theorem 4.3 Assume that $\left(\mathrm{H}_{\phi}\right),\left(\mathrm{H}_{f}\right),\left(\mathrm{H}_{P}\right)$ and $(\mathrm{WN})$ hold. Then periodic problem (1.1), (1.3) has a solution $u \in W(I)$ such that $\alpha(t) \leq u(t) \leq \beta(t)$ for every $t \in I$.

Proof Proposition 4.2 insures the existence of $u \in W(I)$, a solution of $\left(4.1_{\lambda}\right)$ for $\lambda=1$. To conclude, we have to show that $\alpha(t) \leq u(t) \leq \beta(t)$ for every $t \in I$, since $\widehat{f}(t, x, y)=f(t, x, y)$ for $x \in[\alpha(t), \beta(t)]$.

Using $\left(\mathrm{H}_{P}\right)$, we obtain that

$$
\begin{array}{ll}
\left(\phi\left(u^{\prime}(t)\right)\right)^{\prime}<\left(\phi\left(\alpha^{\prime}(t)\right)\right)^{\prime} & \text { a.e. } t \in\{t \in I: u(t)<\alpha(t)\}, \\
\left(\phi\left(u^{\prime}(t)\right)\right)^{\prime}>\left(\phi\left(\beta^{\prime}(t)\right)\right)^{\prime} & \text { a.e. } t \in\{t \in I: u(t)>\beta(t)\} .
\end{array}
$$

It follows from Lemma 2.7 that $\alpha(t) \leq u(t) \leq \beta(t)$ for all $t \in I$.

\section{The Neumann problem}

In this section, we consider problem (1.1) with the Neumann boundary condition. In order to establish the existence of a solution to (1.1), (1.3), we consider the following family of problems defined for $\lambda \in[0,1]$ :

$$
\begin{aligned}
& \left(\phi\left(u^{\prime}(t)\right)\right)^{\prime}=\lambda \tilde{f}\left(t, u(t), u^{\prime}(t)\right)+\frac{(1-\lambda)}{T} \int_{0}^{T} \tilde{f}\left(t, u(t), u^{\prime}(t)\right) d t \quad \text { a.e. } t \in I, \\
& \phi\left(u^{\prime}(0)\right)=\mu, \quad \phi\left(u^{\prime}(T)\right)=v
\end{aligned}
$$

where $\widetilde{f}:[0,1] \times \mathbb{R}^{2} \rightarrow \mathbb{R}$ is defined by

$$
\tilde{f}(t, x, y)= \begin{cases}f\left(t, \beta(t), \beta^{\prime}(t)\right)-\widetilde{h}(t)(x-\beta(t)) & \text { if } x>\beta(t), \\ f(t, x, y) & \text { if } \alpha(t) \leq x \leq \beta(t), \\ f\left(t, \alpha(t), \alpha^{\prime}(t)\right)+\widetilde{g}(t)(x-\alpha(t)) & \text { if } x<\alpha(t)\end{cases}
$$

with $\widetilde{g}, \widetilde{h} \in L^{1}(I)$ chosen such that

$$
\begin{aligned}
& \tilde{g}(t)>\max \left\{0, f\left(t, \alpha(t), \alpha^{\prime}(t)\right)-\frac{1}{T}(v-\mu)\right\}, \\
& \tilde{h}(t)<\min \left\{0, f\left(t, \beta(t), \beta^{\prime}(t)\right)-\frac{1}{T}(v-\mu)\right\} .
\end{aligned}
$$

We show that the solutions to these problems are a priori bounded. Let $w \in \mathcal{C}^{1}(I)$ and $v \in W(I)$ be defined by

$$
w(t)=\mu+\frac{t}{T}(v-\mu) \quad \text { and } \quad v=\Phi(0, w) .
$$


Observe that

$$
\phi\left(v^{\prime}\right)=w .
$$

Proposition 5.1 Assume that $\left(\mathrm{H}_{\phi}\right),\left(\mathrm{H}_{f}\right),\left(\mathrm{H}_{N}\right)$ and $(\mathrm{WN})$ hold. Then there exists $M>$ $\left\|v^{\prime}\right\|_{0}$ such that any solution $u$ of $\left(5.1_{\lambda}\right)$ satisfies $\|u-v\|_{0}<M$ and $\left\|u^{\prime}\right\|_{0}<M$. Moreover,

$$
\left\{u \in \mathcal{C}^{1}(I): \alpha(t) \leq u(t) \leq \beta(t) \forall t \in I\right\} \subset\left\{u \in \mathcal{C}^{1}(I):\|u-v\|_{0}<M\right\}
$$

Proof Fix $M_{1}>0$ such that

$$
-M_{1}+v(t) \leq \min _{t \in I} \alpha(t)-1 \quad \text { and } \quad M_{1}+v(t) \geq \max _{t \in I} \beta(t)+1 \quad \forall t \in I .
$$

We claim that any solution $u$ of $\left(5.1_{\lambda}\right)$ is such that $\|u-v\|_{0} \leq M_{1}$. For every $u \in W(I)$, one has almost everywhere on $\left\{t \in I: u(t)<-M_{1}+v(t)\right\}$,

$$
\begin{aligned}
\tilde{f}\left(t, u(t), u^{\prime}(t)\right) & =f\left(t, \alpha(t), \alpha^{\prime}(t)\right)+\widetilde{g}(t)(u(t)-\alpha(t)) \\
& <f\left(t, \alpha(t), \alpha^{\prime}(t)\right)-\widetilde{g}(t) \\
& \leq \frac{1}{T}(v-\mu) .
\end{aligned}
$$

Similarly,

$$
\widetilde{f}\left(t, u(t), u^{\prime}(t)\right)>\frac{1}{T}(v-\mu) \quad \text { a.e. on }\left\{t \in I: u(t)>M_{1}+v(t)\right\}
$$

Let $u$ be a solution of $\left(5.1_{\lambda}\right)$. One has

$$
v-\mu=\int_{0}^{T}\left(\phi\left(u^{\prime}(t)\right)\right)^{\prime} d t=\int_{0}^{T} \tilde{f}\left(t, u(t), u^{\prime}(t)\right) d t .
$$

Combining (5.5), (5.6) and (5.7), we deduce that almost everywhere on $\left\{t \in I: u(t)<-M_{1}+\right.$ $v(t)\}$,

$$
\begin{aligned}
\left(\phi\left(u^{\prime}(t)\right)\right)^{\prime} & =\lambda \tilde{f}\left(t, u(t), u^{\prime}(t)\right)+\frac{(1-\lambda)}{T}(v-\mu) \\
& \leq \frac{1}{T}(v-\mu) \\
& =\left(\phi\left(v^{\prime}(t)\right)\right)^{\prime} .
\end{aligned}
$$

Similarly,

$$
\left(\phi\left(u^{\prime}(t)\right)\right)^{\prime} \geq\left(\phi\left(v^{\prime}(t)\right)\right)^{\prime} \quad \text { a.e. on }\left\{t \in I: u(t)>M_{1}+v(t)\right\} .
$$

Moreover,

$$
\phi\left(u^{\prime}(0)\right)=\mu=\phi\left(v^{\prime}(0)\right) \text { and } \phi\left(u^{\prime}(T)\right)=v=\phi\left(v^{\prime}(T)\right) \text {. }
$$


It follows from Lemma 2.7 that $\|u-v\|_{0} \leq M_{1}$, or there exists $c>0$ such that $|u(t)-v(t)|=$ $M_{1}+c$ for all $t \in I$. If $u(t)=v(t)+M_{1}+c$ for all $t \in I$, then

$$
\begin{aligned}
v-\mu & =\int_{0}^{T} \tilde{f}\left(t, u(t), u^{\prime}(t)\right) d t \\
& =\int_{0}^{T} f\left(t, \beta(t), \beta^{\prime}(t)\right)-\tilde{h}(t)\left(v(t)+M_{1}+c-\beta(t)\right) d t \\
& >\int_{0}^{T}\left(\phi\left(\beta^{\prime}(t)\right)\right)^{\prime} d t \\
& =\phi\left(\beta^{\prime}(T)\right)-\phi\left(\beta^{\prime}(0)\right) \\
& \geq v-\mu
\end{aligned}
$$

a contradiction. Similarly, one cannot have $u(t)=v(t)-M_{1}-c$ for all $t \in I$. Hence, $\| u-$ $v \|_{0} \leq M_{1}$.

Let

$$
\widetilde{l}_{1}(t)=\max \left\{\left|f\left(t, \alpha(t), \alpha^{\prime}(t)\right)\right|+2 M_{1}|\widetilde{g}(t)|,\left|f\left(t, \beta(t), \beta^{\prime}(t)\right)\right|+2 M_{1}|\widetilde{h}(t)|\right\} .
$$

Observe that, by (WN), one has that for any $u$ solution of $\left(5.1_{\lambda}\right)$,

$$
\begin{aligned}
\left|\left(\phi\left(u^{\prime}(t)\right)\right)^{\prime}\right| & \leq \max \left\{\widetilde{l}_{1}(t), \psi\left(\left|u^{\prime}(t)\right|\right)\left(l(t)+c(t)\left|u^{\prime}(t)\right|^{(p-1) / p}\right)\right\} \\
& \left.\leq \psi\left(\left|u^{\prime}(t)\right|\right) \widetilde{l}_{0}(t)+c(t)\left|u^{\prime}(t)\right|^{(p-1) / p}\right) \quad \text { a.e. } t \in I
\end{aligned}
$$

with

$$
\tilde{l}_{0}(t)=\max \left\{l(t), \frac{\tilde{l}_{1}(t)}{k}\right\} .
$$

It follows from Lemma 2.9 applied with $d_{0}=\phi^{-1}(\mu)$ that there exists $M_{0}$ such that any solution $u$ of $\left(5.1_{\lambda}\right)$ satisfies $\left\|u^{\prime}\right\|_{0}<M_{0}$.

Finally, set $M=\max \left\{M_{0}, 1+M_{1}, 1+\left\|v^{\prime}\right\|_{0}\right\}$, we get the conclusion.

Proposition 5.2 Assume that $\left(\mathrm{H}_{\phi}\right),\left(\mathrm{H}_{f}\right),\left(\mathrm{H}_{N}\right)$ and $(\mathrm{WN})$ hold. Then, for every $\lambda \in[0,1]$, problem $\left(5.1_{\lambda}\right)$ has at least one solution.

Proof Let $N_{\tilde{f}}: \mathcal{C}^{1}(I) \rightarrow \mathcal{C}(I), \widetilde{L}: \mathcal{C}^{1}(I) \rightarrow \mathcal{C}(I)$ and $\mathcal{S}_{N}:[0,1] \times \mathcal{C}^{1}(I) \rightarrow \mathbb{R} \times \mathcal{C}(I)$ be defined respectively by

$$
\begin{aligned}
& N_{\tilde{f}}(u)(t)=\int_{0}^{t} \tilde{f}\left(s, u(s), u^{\prime}(s)\right) d s, \\
& \widetilde{L}(u)(t)=\frac{t}{T} N_{\tilde{f}}(u)(T), \\
& \mathcal{S}_{N}(\lambda, u)=\left(u(0)+\frac{1}{T}\left(\mu-v+N_{\tilde{f}}(u)(T)\right), w+\lambda\left(N_{\tilde{f}}(u)-\widetilde{L}(u)\right)\right),
\end{aligned}
$$


with $w \in \mathcal{C}^{1}(I)$ defined in (5.4). Now, we define $\mathcal{N}:[0,1] \times \mathcal{C}^{1}(I) \rightarrow \mathcal{C}^{1}(I)$ by

$$
\mathcal{N}=\Phi \circ \mathcal{S}_{N}
$$

Again, we deduce from Lemmas 2.4, 2.5 and 2.6 that $\mathcal{S}_{N}$ and $\mathcal{N}$ are continuous and completely continuous.

Now, we study the fixed points of $\mathcal{N}$. Let $u \in \mathcal{C}^{1}(I)$ and $\lambda \in[0,1]$ be such that $u=\mathcal{N}(\lambda, u)$. One has

$$
u(0)=u(0)+\frac{1}{T}\left(\mu-v+N_{\tilde{f}}(u)(T)\right) .
$$

So,

$$
v-\mu=N_{\tilde{f}}(u)(T)
$$

Also, $\phi\left(u^{\prime}\right)=w+\lambda\left(N_{\tilde{f}}(u)-\widetilde{L}(u)\right)$. Hence, by Lemma 2.4 , it is absolutely continuous and

$$
\begin{aligned}
\left(\phi\left(u^{\prime}(t)\right)\right)^{\prime} & =\lambda\left(\tilde{f}\left(t, u(t), u^{\prime}(t)\right)-\frac{1}{T} N_{\tilde{f}}(u)(T)\right)+\frac{1}{T}(v-\mu) \\
& =\lambda \tilde{f}\left(t, u(t), u^{\prime}(t)\right)+\frac{(1-\lambda)}{T} \int_{0}^{T} \tilde{f}\left(t, u(t), u^{\prime}(t)\right) d t \quad \text { a.e. } t \in I .
\end{aligned}
$$

Moreover,

$$
\begin{aligned}
& \phi\left(u^{\prime}(0)\right)=w(0)+\lambda\left(N_{\tilde{f}}(u)(0)-\widetilde{L}(u)(0)\right)=\mu, \\
& \phi\left(u^{\prime}(T)\right)=w(T)+\lambda\left(N_{\tilde{f}}(u)(T)-\widetilde{L}(u)(T)\right)=v .
\end{aligned}
$$

So, fixed points of $\mathcal{N}$ are solutions of $\left(5.1_{\lambda}\right)$.

Let $M>0$ be the constant obtained in Proposition 5.1 and $v=\Phi(0, w)$. We set

$$
\mathcal{U}=\left\{u \in \mathcal{C}^{1}(I):\|u-v\|_{0}<M,\left\|u^{\prime}\right\|_{0}<M\right\} .
$$

Proposition 5.1 implies that $u \neq \mathcal{N}(\lambda, u)$ for all $(\lambda, u) \in[0,1] \times \partial \mathcal{U}$. By the homotopy property of the fixed point index,

$$
\operatorname{index}(\mathcal{N}(\lambda, \cdot), \mathcal{U})=\operatorname{index}(\mathcal{N}(0, \cdot), \mathcal{U}) \quad \forall \lambda \in[0,1]
$$

Observe that

$$
\mathcal{N}(0, u)=u(0)+\frac{1}{T}\left(\mu-v+N_{\tilde{f}}(u)(T)\right)+\Phi(0, w) \in \mathbb{R}+v .
$$

Let $X=\{u=r+v: r \in \mathbb{R}\} \subset \mathcal{C}^{1}(I)$. Notice that $X$ is not a normed vectorial space if $v \neq 0$. Nevertheless, if $v \neq 0, X$ is an affine space and hence it is an ANR. By the contraction property of the fixed point index (see [18, Chapter 4, Section 12 , Theorem 6.2]),

$$
\operatorname{index}(\mathcal{N}(0, \cdot), \mathcal{U})=\operatorname{index}(\mathcal{N}(0, \cdot), \mathcal{U} \cap X)
$$


One has $\partial(\mathcal{U} \cap X)=\{-M+v, M+v\}$. Consider $H:[-M, M] \rightarrow \mathbb{R}$ defined by

$$
H(r)=\mathcal{N}(0, r+v)-v .
$$

By (5.6),

$$
H(M)=\mathcal{N}(0, M+v)-v=M+\frac{1}{T}\left(\mu-v+\int_{0}^{T} \tilde{f}\left(t, M+v(t), v^{\prime}(t)\right) d t\right)>M,
$$

since $M+v(t)>\beta(t)$ for every $t \in I$. Similarly,

$$
H(-M)<-M \text {. }
$$

By the commutativity property of the fixed point index (see [18, Chapter 4 , Section 12 , Theorem 6.2]),

$$
\operatorname{index}(\mathcal{N}(0, \cdot), \mathcal{U} \cap X)=\operatorname{index}(H,]-M, M[)=-1 .
$$

Combining (5.11), (5.12) and (5.13), we deduce that for every $\lambda \in[0,1]$,

$$
\operatorname{index}(\mathcal{N}(\lambda, \cdot), \mathcal{U})=-1
$$

Thus, $\mathcal{N}(\lambda, \cdot)$ has a fixed point, and hence $\left(5.1_{\lambda}\right)$ has a solution.

Now, we can establish the existence of a solution to (1.1), (1.4).

Theorem 5.3 Assume that $\left(\mathrm{H}_{\phi}\right),\left(\mathrm{H}_{f}\right),\left(\mathrm{H}_{N}\right)$ and $(\mathrm{WN})$ hold. Then Neumann problem (1.1), (1.4) has a solution $u \in W(I)$ such that $\alpha(t) \leq u(t) \leq \beta(t)$ for every $t \in I$.

Proof Proposition 5.2 insures the existence of $u \in W(I)$, a solution of $\left(5.1_{\lambda}\right)$ for $\lambda=1$. To conclude, we have to show that $\alpha(t) \leq u(t) \leq \beta(t)$ for every $t \in I$, since $\widetilde{f}(t, x, y)=f(t, x, y)$ for $x \in[\alpha(t), \beta(t)]$.

Using $\left(\mathrm{H}_{N}\right)$, we obtain that

$$
\begin{array}{ll}
\left(\phi\left(u^{\prime}(t)\right)\right)^{\prime}<\left(\phi\left(\alpha^{\prime}(t)\right)\right)^{\prime} & \text { a.e. } t \in\{t \in I: u(t)<\alpha(t)\}, \\
\left(\phi\left(u^{\prime}(t)\right)\right)^{\prime}>\left(\phi\left(\beta^{\prime}(t)\right)\right)^{\prime} & \text { a.e. } t \in\{t \in I: u(t)>\beta(t)\} .
\end{array}
$$

It follows from Lemma 2.7 that $\alpha(t) \leq u(t) \leq \beta(t)$ for all $t \in I$.

\section{Multiplicity result}

In this section, we establish the existence of at least three solutions to problem (1.1).

Theorem 6.1 Assume that $\left(\mathrm{H}_{\phi}\right),\left(\mathrm{H}_{f}\right)$ and the following conditions are satisfied:

$\left(\mathrm{H}_{\mathcal{B}}\right)^{\prime}$ For $i=1,2$, there exist $\alpha_{i}, \beta_{i} \in W(I)$, respectively strict lower and upper solutions of (1.1), such that $\alpha_{i}(t)<\beta_{i}(t), \alpha_{1}(t) \leq \alpha_{2}(t), \beta_{1}(t) \leq \beta_{2}(t)$ for all $t \in I$, and $\left\{t \in I: \alpha_{2}(t)>\right.$ $\left.\beta_{1}(t)\right\} \neq \emptyset$. 
$(\mathrm{WN})^{\prime}$ There exist $\left.\left.k>0, p \in\right] 1, \infty\right], c \in L^{p}\left(I,\left[0, \infty[), l \in L^{1}(I,[0, \infty[)\right.\right.$ and $\psi:[0, \infty[\rightarrow$ $[k, \infty[$ such that

$$
\frac{1}{\psi \circ \phi^{-1}} \in L_{l o c}^{1}(\mathbb{R}), \quad \int_{0}^{ \pm \infty} \frac{d s}{\psi\left(\left|\phi^{-1}(s)\right|\right)}= \pm \infty,
$$

and

$$
\begin{aligned}
& |f(t, x, y)| \leq \psi(|y|)\left(l(t)+c(t)|y|^{(p-1) / p}\right) \\
& \quad \forall x \in\left[\alpha_{1}(t), \beta_{2}(t)\right], y \in \mathbb{R} \text {, and a.e. } t \in I,
\end{aligned}
$$

with $(p-1) / p=1$ if $p=\infty$.

Then problem (1.1) has at least three solutions $u_{1}, u_{2}, u_{3}$ such that

$$
\alpha_{1}(t)<u_{3}(t)<\beta_{2}(t), \quad \alpha_{i}(t)<u_{i}(t)<\beta_{i}(t) \quad \forall t \in I, \text { and } i=1,2,
$$

and $\left\{t \in I: \beta_{1}(t) \leq u_{3}(t) \leq \alpha_{2}(t)\right\} \neq \emptyset$.

Proof Let $\mathcal{B}$ denote Dirichlet boundary condition (1.2). Consider $\mathcal{D}_{1}, \mathcal{D}_{2}$ and $\mathcal{D}_{3}$, the operators defined as in (3.7) and associated to the pairs of lower and upper solutions $\left(\alpha_{1}, \beta_{1}\right)$, $\left(\alpha_{2}, \beta_{2}\right)$ and $\left(\alpha_{1}, \beta_{2}\right)$, respectively. Let $\mathcal{U}_{i} \subset \mathcal{C}^{1}(I)$ be the open set defined as in (3.8) and associated to the operator $\mathcal{D}_{i}$ for $i=1,2,3$. From equation (3.9), one has

$$
\operatorname{index}\left(\mathcal{D}_{i}(1, \cdot), \mathcal{U}_{i}\right)=1 \quad \text { for } i=1,2,3 \text {. }
$$

From the proof of Theorem 3.3, we deduce that any fixed point $u$ of $\mathcal{D}_{i}(1, \cdot)$ is a solution of (1.1), (1.2) and is such that $\alpha_{i}(t) \leq u(t) \leq \beta_{i}(t)$ for all $t \in I$ if $i=1,2$, and such that $\alpha_{1}(t) \leq$ $u(t) \leq \beta_{2}(t)$ for all $t \in I$ if $i=3$. Using the fact that $\alpha_{i}$ and $\beta_{i}$ are respectively strict lower and upper solutions of (1.1), it follows from Lemma 2.8 that $\mathcal{D}_{i}(1, \cdot)$ has no fixed points in $\mathcal{U}_{i} \backslash \overline{\mathcal{V}_{i}}$, with

$$
\begin{aligned}
& \mathcal{V}_{i}=\left\{u \in \mathcal{C}^{1}(I): \alpha_{i}(t)<u(t)<\beta_{i}(t) \forall t \in I\right\} \quad \text { for } i=1,2, \\
& \mathcal{V}_{3}=\left\{u \in \mathcal{C}^{1}(I): \alpha_{1}(t)<u(t)<\beta_{2}(t) \forall t \in I\right\} .
\end{aligned}
$$

Hence, by the excision property of the fixed point index,

$$
\operatorname{index}\left(\mathcal{D}_{i}(1, \cdot), \mathcal{V}_{i}\right)=1 \quad \text { for } i=1,2,3 \text {. }
$$

Since $\alpha_{1}(t) \leq \beta_{1}(t) \leq \beta_{2}(t), \alpha_{1}(t) \leq \alpha_{2}(t) \leq \beta_{2}(t)$ for all $t \in I$ and $\left\{t \in I: \alpha_{2}(t)>\beta_{1}(t)\right\} \neq \emptyset$, one has

$$
\mathcal{V}_{1} \cup \mathcal{V}_{2} \subset \mathcal{V}_{3}, \quad \text { and } \quad \mathcal{V}_{3} \backslash\left(\overline{\mathcal{V}_{1} \cup \mathcal{V}_{2}}\right) \neq \emptyset \text {, }
$$

and

$$
\mathcal{D}_{i}(1, u)=\mathcal{D}_{3}(1, u) \quad \forall u \in \mathcal{V}_{i}, i=1,2
$$


This combined with the additivity of the fixed point index implies that

$$
\begin{aligned}
\operatorname{index}\left(\mathcal{D}_{3}(1, \cdot), \mathcal{V}_{3} \backslash\left(\overline{\mathcal{V}_{1} \cup \mathcal{V}_{2}}\right)\right)= & \operatorname{index}\left(\mathcal{D}_{3}(1, \cdot), \mathcal{V}_{3}\right) \\
& -\operatorname{index}\left(\mathcal{D}_{3}(1, \cdot), \mathcal{V}_{1}\right)-\operatorname{index}\left(\mathcal{D}_{3}(1, \cdot), \mathcal{V}_{2}\right) \\
= & -1 .
\end{aligned}
$$

Therefore, problem (1.1) has at least three solutions $u_{1} \in \mathcal{V}_{1}, u_{2} \in \mathcal{V}_{2}$ and $u_{3} \in \mathcal{V}_{3} \backslash\left(\overline{\mathcal{V}_{1} \cup \mathcal{V}_{2}}\right)$. We argue similarly if $\mathcal{B}$ denotes periodic boundary condition (1.3) (resp. Neumann boundary condition (1.4)) by using the results of Section 4 (resp. Section 5).

\section{Competing interests}

The authors declare that they have no competing interests.

\section{Authors' contributions}

Authors contributed equally. They read and approved the final manuscript.

\section{Author details}

${ }^{1}$ Département de mathématiques, Faculté des sciences de Rabat, Université Mohammed V, Agdal, Rabat, Marocco. ${ }^{2}$ Département de mathématiques et de statistique, Université de Montréal, succ. Centre-Ville, C.P. 6128, Montréal, H3C 3J7, Canada.

Received: 23 May 2013 Accepted: 4 October 2013 Published: 08 Nov 2013

\section{References}

1. Ben-Naoum, AK, De Coster, C: On the existence and multiplicity of positive solutions of the $p$-Laplacian separated boundary value problem. Differ. Integral Equ. 10, 1093-1112 (1997)

2. Bereanu, C, Mawhin, J: Multiple periodic solutions of ordinary differential equations with bounded nonlinearities and $\phi$-Laplacian. NoDEA Nonlinear Differ. Equ. Appl. 15, 159-168 (2008)

3. Cabada, A, Pouso, RL: Existence result for the problem $\left(\phi\left(u^{\prime}\right)\right)^{\prime}=f\left(t, u, u^{\prime}\right)$ with periodic and Neumann boundary conditions. Nonlinear Anal. 30, 1733-1742 (1997)

4. Cabada, A, Pouso, RL: Existence results for the problem $\left(\phi\left(u^{\prime}\right)\right)^{\prime}=f\left(t, u, u^{\prime}\right)$ with nonlinear boundary conditions. Nonlinear Anal. 35, 221-231 (1999)

5. Cabada, A, Pouso, RL: Existence theory for functional $p$-Laplacian equations with variable exponents. Nonlinear Anal. 52, 557-572 (2003)

6. Cabada, A, O'Regan, D, Pouso, RL: Second order problems with functional conditions including Sturm-Liouville and multipoint conditions. Math. Nachr. 281, 1254-1263 (2008)

7. De Coster, C: On pairs of positive solutions for the one dimensional p-Laplacian. Nonlinear Anal. 23, 669-681 (1994)

8. El Khattabi, N: Problèmes périodiques du second ordre à croissance au plus linéaire. Topol. Methods Nonlinear Anal. 5, 365-383 (1995)

9. Ferracuti, L, Papalini, F: Boundary-value problems for strongly non-linear multivalued equations involving different $\phi$-Laplacians. Adv. Differ. Equ. 14, 541-566 (2009)

10. Frigon, M: Théorèmes d'existence de solutions d'inclusions différentielles. In: Topological Methods in Differential Equations and Inclusions (Montreal, 1994). NATO Adv. Sci. Inst. Ser. C Math. Phys. Sci., vol. 472, pp. 51-87. Kluwer Academic, Dordrecht (1995)

11. Goudreau, K: A multiplicity result for a nonlinear boundary value problem. J. Math. Anal. Appl. 218, $395-408$ (1998)

12. Mawhin, J, Thompson, HB: Nagumo conditions and second-order quasilinear equations with compatible nonlinear functional boundary conditions. Rocky Mt. J. Math. 41, 573-596 (2011)

13. O'Regan, D: Some general existence principles and results for $\left(\phi\left(y^{\prime}\right)\right)^{\prime}=q(t) f\left(t, y, y^{\prime}\right), 0<t<1$. SIAM J. Math. Anal. 24, 648-668 (1993)

14. Wang, J, Gao, W, Lin, Z: Boundary value problems for general second order equations and similarity solutions to the Rayleigh problem. Tohoku Math. J. 45, 327-344 (1995)

15. Wang, J, Gao, W: Existence of solutions to boundary value problems for a nonlinear second order equation with weak Carathéodory functions. Differ. Equ. Dyn. Syst. 5, 175-185 (1997)

16. Zhang, JJ, Liu, WB, Ni, JB, Chen, TY: Multiple periodic solutions of $p$-Laplacian equation with one-side Nagumo condition. J. Korean Math. Soc. 45, 1549-1559 (2008)

17. Manásevich, R, Mawhin, J: Periodic solutions for nonlinear systems with p-Laplacian-like operators. J. Differ. Equ. 145, 367-393 (1998)

18. Granas, A, Dugundji, J: Fixed Point Theory. Springer Monographs in Mathematics. Springer, New York (2003)

10.1186/1687-2770-2013-236

Cite this article as: El Khattabi et al.: Multiple solutions of boundary value problems with $\phi$-Laplacian operators and under a Wintner-Nagumo growth condition. Boundary Value Problems 2013, 2013:236 